\title{
Routing, Spectrum, and Transponder Assignment in Elastic Optical Networks
}

\author{
M. Dallaglio, A. Giorgetti, N. Sambo, L. Velasco, and P. Castoldi
}

\begin{abstract}
Backbone networks are evolving toward elastic optical network (EON) architecture that allows a flexible and efficient use of spectrum resources. Flexibility in EONs is also guaranteed by emerging sliceable bandwidth variable transponders (SBVTs) that support the simultaneous generation of multiple optical carriers. Such carriers can be used to serve different lightpaths (i.e., slice-ability), or can be merged into a single high-rate superchannel. SBVTs typically use a dedicated laser to generate each carrier, i.e., multilasers SBVT (ML-SBVT). Alternatively, a multiwavelength source can be used to generate multiple carriers using a single laser, i.e., multiwavelength SBVT (MW-SBVT). MW-SBVT improves the super-channel spectrum efficiency. Indeed, MW-SBVT reduces the intercarrier interference among the subcarriers composing the super-channel; thus, it is possible to reduce the guard bands among subcarriers. With ML-SBVT, each subcarrier suffers from unstableness of the related laser and intercarrier interference may have a huge impact, thus, higher guard bands are needed. On the other hand, the use of a MWSBVT introduces specific constraints to the routing and spectrum assignment (RSA), because the spacing among the subcarriers is limited to a range of specific values. To take into account the constraints introduced by transponders, this paper integrates the selection of the transponder into RSA; thus, proposing a dynamic routing, spectrum, and transponder assignment (RSTA) scheme supporting both ML-SBVT and MW-SBVT technologies, and aiming to combine the benefits of the two technologies. Simulation results show that the proposed RSTA scheme provides benefits in terms of achieved blocking probability compared to the traditional RSA schemes. Moreover, the achieved results demonstrate that jointly using both SBVT technologies provides significant benefits with respect to the utilization of any single SBVT technology.
\end{abstract}

Index Terms-Multi-lasers, multi-wavelength, RSTA, SBVT, slice-ability.

\section{INTRODUCTION}

B ACKBONE networks are gradually evolving to enable a more efficient utilization of the spectrum provided by the optical fibers. To this extent, elastic optical networks (EONs) have been recently introduced. In EONs, the spectrum is exploited by means of a flexible grid and end-to-end optical connections (i.e., lightpaths) occupy a portion of the spectrum

Manuscript received April 22, 2015; revised September 7, 2015; accepted September 9, 2015. Date of publication September 10, 2015; date of current version October 5, 2015. This paper is an extended version of the work presented at the Optical Fiber and Communication Conference, Los Angeles, CA, USA, Mar. 2015 [1]. This work was supported in part by the EU-FP7 IDEALIST project.

M. Dallaglio, A. Giorgetti, N. Sambo, and P. Castoldi are with the Scuola Superiore Sant'Anna, Pisa 56100, Italy (e-mail: m.dallaglio@sssup.it; alessio.giorgetti@sssup.it; n.sambo@sssup.it; castoldi@sssup.it).

L. Velasco is with the Universitat Politcnica de Catalunya, Barcelona 08034, Spain (e-mail: lvelasco@ac.upc.edu).

Color versions of one or more of the figures in this paper are available online at http://ieeexplore.ieee.org.

Digital Object Identifier 10.1109/JLT.2015.2477898 whose width depends on traffic requirements and transmission parameters such as bitrate and modulation format [2]. Thus, typically, lightpaths with different spectrum utilization are contemporarily active in EONs [3], [4].

Further flexibility is achieved in EONs with the introduction of multi-flow transponders or sliceable bandwidth variable transponders (SBVTs). SBVTs support slice-ability, i.e., the capability of generating multiple optical carriers that can be used to support different lightpaths towards different destinations, or can be merged into a single high-rate superchannel. This concept were originally introduced in [2], and, in the last years, they have been deeply investigated, spanning from the presentation of the SBVT architecture [5], [6] (including the electronics layer based on the optical transport network (OTN) standard [7]) to the optical carriers generation techniques (e.g., array of lasers) [6], [8], power-budget and techno-economical analysis [9]-[11], network performance evaluation [12], comparison of several transmission techniques [13], [14], and, finally, the experimental demonstration of SBVT [8], [15], [16]. A widely agreed conclusion is that SBVTs and slice-ability are attractive features for operators because of the provided flexibility in terms of programmable rate per destinations, cost reduction when migrating towards high rate super-channels, and prospects for the integrability of several transponder elements into a single chip [13].

Regarding the generation of the optical carriers, two technologies can be adopted. Typically, SBVTs use a dedicated tunable laser to generate each carrier. We refer to this technology as multi-lasers SBVT (i.e., ML-SBVT). Alternatively, a multi-wavelength source (i.e., a source able to generate several optical carriers from a single laser) can be used [6], [8], [17]. We refer to this technology as multi-wavelength SBVT (i.e., MW-SBVT). MW-SBVT provides several benefits but, at the same time, it introduces transponder specific constraints to the RSA problem [18], [19]. Specifically, the super-channel spectrum efficiency is improved using MW-SBVTs because, by using a single laser source, optical carriers are intrinsically locked, therefore within a super-channel the guard bands between carriers can be significantly reduced. Conversely, whereas MLSBVT provides full and independent tunability of each optical carrier, MW-SBVT fully supports the tunability of the whole comb but the spacing among carriers is limited by a maximum value [6], [17]. Therefore, when establishing a new lightpath using a MW-SBVT, the applied RSA has to carefully consider the transponder to be selected because some of them, although partially available, could be unable to support some portions of spectrum due to intrinsic tunability limitation. As an example, if only one carrier is used in a MW-SBVT and allocated to a 
specific portion of spectrum, all the other carriers generated by this transponder are constrained to be used within a specific range of spectrum around the spectrum allocated to the first carrier.

Several research works investigated the network performance achievable by EONs, however, more effort is required to understand the impact of MW-SBVTs on EON performance. Specifically, both static and dynamic RSA problems have been studied in the EON scenario where the issues introduced by spectrum fragmentation have to be carefully considered [20]. Static RSA algorithms are applied offline during network design and planning, they typically exploit ILP formulations [21], however a number of heuristic algorithms have been also proposed [18], [22]-[25]. Dynamic RSA algorithms are applied during network operation when new lightpaths have to be established. The work in [26] studied dynamic RSA algorithms under different grid spacings proposing a specific allocation scheme; Wan et al. [27] proposed two different RSA heuristics based on the problem decomposition approach; Beyranvand and Salehi [28] and Sambo et al. [29] included the evaluation of quality of transmission constraint within some RSA heuristics. Other works focused on spectrum fragmentation management by applying specifically designed dynamic RSA algorithms [30]-[32] and also consider the possibility to periodically re-optimize the link spectrum utilization [33], [34].

Several previous works in [12], [35]-[38] considered the application of slice-ability in a dynamic traffic scenario. In these studies, when a lightpath request cannot be allocated as a single super-channel, slice-ability is used to split the request in a number of sub-lightpaths that are independently routed in the network and each one has to fit the ITU-T flex-grid. This introduces a resource overbuild with respect to the utilization of a single super-channel. However our previous work in [35] and [12] demonstrated that, using ML-SBVTs, sliceability may increases the network utilization (i.e., reducing the achievable lightpath blocking probability) despite the introduction the aforementioned resource overbuild. An investigation of slice-ability impact using MW-SBVT has been performed in [39] considering an unlimited number of transponders per node. The achieved results demonstrated that MW-SBVTs provide benefits with respect to ML-SBVTs only if slice-ability is not allowed. Moreover, the potential benefits of slice-ability have been also studied in a static traffic scenario where it can provide significant cost savings to the network provider [9].

When applying slice-ability the several sub-channels can also be allowed to use different paths. This increases the slice-ability effectiveness but it introduces differential delay between subchannels that has to be compensated at the destination node. The studies in [36] and [37] compare single-path and multi-path slice-ability, whereas the work in [40] focuses on a GMPLS control plane extension to support multi-path slice-ability. Differential delay compensation is typically demanded at the OTN layer that natively support Virtual Concatenation [7]. Moreover, several research works proposed methods to effectively perform delay compensation [41], [42].

This paper evaluates the performance of ML-SBVT and MWSBVT technologies in EON network scenario with a limited number of transponders per node. Network performance is investigated as a function of the number of available transponders per node and as a function of the offered network load. A dynamic traffic scenario is considered where lightpaths requiring different bandwidth are established and released as time evolves. Thus, independently on the network load, lightpaths with different spectrum occupation are contemporarily active in the network. In this scenario, a novel dynamic routing, spectrum, and transponder assignment (i.e., RSTA) scheme is proposed that integrates the selection of the transponder with the RSA accounting for the knowledge of the transponder technology, their current availability, and the constraints introduced by each specific transponder. First, RSTA is run to quantify the effects of the improved spectral efficiency achieved by MW-SBVTs used to serve high-rate super-channels. Then, slice-ability is evaluated by comparing MW-SBVT and ML-SBVT technologies. Results show that MW-SBVT is more effective to serve superchannels, whereas ML-SBVT provides significant benefit when slice-ability is applied. Finally, we consider the possibility to combine the benefits of both technologies in a heterogeneous scenario where each network node is equipped with a number of MW-SBVTs and a number of ML-SBVTs. In this heterogeneous scenario we show that a traditional RSA is not able to exploit the potential benefit of both SBVT architecture. Conversely, the proposed RSTA provides significant network performance improvement by effectively assigning a transponder to a specific lightpath request depending on the transponder technology and the lightpath spectrum requirements.

\section{SBVT Multi-Laser AND Multi-WAVELENGTH TECHNOLOGIES}

Focusing on the SBVT technology agreed by several network operators and vendors, SBVT is composed by a number of modules [13]. At the transmitter side, a first module consists on the OTN framer which adds the forward error correction (FEC) and adapts data clients to the optical layer. Then, a switching matrix distributes the encoded data clients to the proper modulators for the modulation of the optical carrier. The sub-carrier generator module generates $N$ unmodulated optical carriers (i.e., sub-carriers). With both considered SBVT architectures we assumed that all the generated carriers are combined through a coupler/splitter before entering the ingress node. Therefore, carriers generated by the same SBVT cannot contemporary use the same portion of spectrum even if they are directed toward different links. In the case of ML-SBVT, the sub-carrier generator module consists in an array of tunable lasers (one laser for each sub-carrier), as shown in Fig. 1(a). In the case of MW-SBVT, this module consists on a multi-wavelength source, as shown in Fig. 1(b). Considering tunable lasers, with a ML-SBVT, the frequency of each sub-carrier can assume any value in the $\mathrm{C}$ band. Thus, in the example of Fig. $1(\mathrm{c}), f_{1}, f_{2}, f_{3}$ (i.e., $B_{1}$ and $B_{2}$ ) can be set to any value depending on spectrum availability and on the spectrum assignment policy. Conversely, with a MWSBVT, the first activated carrier can be tuned on the whole $C$ band [8], [17], but the admitted values of $B_{1}$ and $B_{2}$ are limited and depend on the adopted multi-wavelength source technology. 


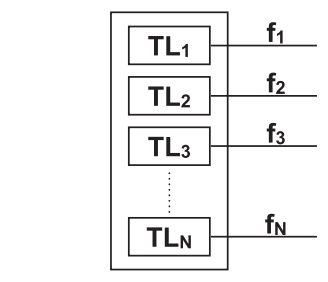

(a)

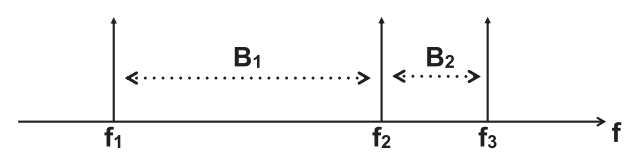

(c)

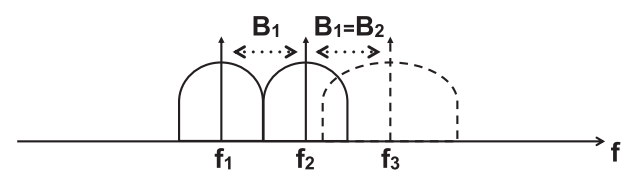

(d)

Fig. 1. Example of sub-carrier generator module in the case of (a) multilaser source, (b) multi-wavelength source, and (c) generated sub-carriers in the spectrum.

Symmetric multi-wavelength sources only admit symmetrical channel spacing $\left(B_{1}=B_{2}\right)$ and are mainly composed of a laser, a Mach-Zehnder modulator, and a radio frequency (RF) source [6], [17]. In this case, the laser provides the parent carrier, which is coupled with the Mach-Zehnder modulator. Then, the sinusoidal RF signal and its double frequency are fed into the modulator. By adjusting the amplitude of the RF signal, an arbitrary number of lines (i.e., 3, 4, 5, or 9) can be generated. The carrier spacing can be adjusted by changing the $\mathrm{RF}$ frequency. The bandwidth of the modulator and of the RF source impose a maximum value $B_{\mathrm{MAX}}$ of channel spacing. All the generated sub-carriers are active at the same time, but not necessary used. Alternatively, asymmetric multi-wavelength sources enable asymmetric channel spacing $\left(B_{1} \neq B_{2}\right)$ and are mainly composed of a laser, several IQ modulators, and RF clocks. Each sub-carrier is obtained by shifting in frequency the parent carrier through the use of a modulator. Each RF clock determines the shift of the parent carrier. With this technology, each sub-carrier can be activated (or not) by activating (or not) its related RF clock which provides the frequency shift. The work reported in [8] demonstrated that a programmable multi-wavelength source with asymmetric channel spacing is technologically feasible. Also in the asymmetric case, $B_{1}$ and $B_{2}$ are limited to a maximum value $B_{\mathrm{MAX}}$ which is given by the bandwidth of the modulators and of the RF clocks. $B_{\text {MAX }}$ can be assumed in the order of $50 \mathrm{GHz}$. Such limitation introduces a constraint in the routing and spectrum assignment (RSA). Moreover, in the case of symmetric multi-wavelength source, consecutive generated sub-carriers are also equally spaced, thus introducing a further constraint during RSA.

Both SBVT architectures require the utilization of guard bands between adjacent lightpaths (i.e., external guard bands) to properly perform switching along the path considering the

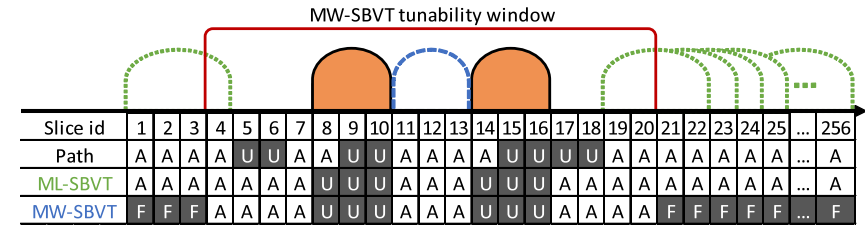

Fig. 2. Example of RSA constraint introduced by a MW-SBVT.

typical resolution of optical filters [43]. Moreover, inside a super-channel, sub-carriers may require guard bands against inter-channel interference. Indeed, the sub-carriers generated by an array of lasers may present instability (typically in the order of 1-2 GHz). Thus, when an ML-SBVT is used to serve a super-channel, those internal guard bands are typically required in order to avoid sub-carrier overlapping within the generated super-channel, resulting in a waste of spectrum [39]. Conversely, within a MW-SBVT the generated sub-carriers are intrinsically locked together, thus when a super-channel has to be served, internal guard bands can be reduced, resulting in a more efficient spectrum utilization. Two independent flows at $f_{1}$ and $f_{2}$ are already in use, as illustrated in Fig. 1(c), when a new lightpath is requested. The same MW-SBVT can be used only if the new request can be accommodated in a sub-carrier whose central frequency is within the range $\left[f_{2}-B_{\mathrm{MAX}} ; f_{2}+B_{\mathrm{MAX}}\right]$ or $\left[f_{1}-B_{\mathrm{MAX}} ; f_{1}+B_{\mathrm{MAX}}\right]$. If it is not possible (e.g., such spectrum is not available along the traversed links), another SBVT has to be used. In the more generic case where up to $N$ carriers are supported and $n$ carriers are already active, a further request has to be accommodated in one of the ranges $f_{n} \pm B_{\text {MAX }}$ where $f_{n}$ is the central frequency of the $n$th active carrier.

We have assumed that the source and the destination transponders are allowed to use a different technology, e.g., given a lightpath connecting a node pair, a ML-SBVT can be used at the transmitter and a MW-SBVT at the receiver.

Fig. 2 explains the RSA constraint introduced by MW-SBVTs illustrating also the spectrum availability along the selected path. According to ITU-T, each lightpath occupies a number of contiguous frequency slices of width $12.5 \mathrm{GHz}$, i.e., the slice id in Fig. 2. An SBVT is considered where two carriers are already allocated, each one serving a lightpath using three frequency slices (i.e., 8-10 and 14-16). Upon arrival of a new $100 \mathrm{~Gb} / \mathrm{s}$ lightpath request, a path is computed with the available slices illustrated in Fig. 2 (i.e., 1-4, 7-8, 11-14, and 19-256). Due to additional internal guard bands required by ML-SBVT, in the example it is assumed that the lightpath occupies a bandwidth of four slices in case of ML-SBVT or three slices in case of MW-SBVT. If a ML-SBVT is used, all the slots supported by the path and large enough four slices can be actually used, except for those already allocated by the transponder. Thus, the lightpath can be established using the slots 1-4, 19-22, 20-23, 21-24 and so on (see dotted in Fig. 2). If a MW-SBVT is used, none of the available slots that fall outside the tunability window can be used because of the limited tunability among generated carriers. Indeed, if the maximum sub-carrier spacing allowed by the MW-SBVT is $50 \mathrm{GHz}$ (i.e., four frequency slices) the slots 1-3, 21-256 are forbidden. However, the higher spectral 
efficiency provided by the MW-SBVT allows to accommodate the request in the empty slot 11-13 (see dashed in Fig. 2).

To specify the constraint introduced by a symmetric MW source we refer to Fig. 2 assuming a path in which all the frequency slices are available. This way, if an asymmetric MW source is used, the third carrier (occupying three slices) can be activated within the tunability window using slots 4-6, 5-7, 11$13,17-19$, or 18-20. Conversely, if a symmetric MW source is adopted, only slot $11-13$ can be used, since it is the only assignment guaranteeing a symmetrical channel spacing. Symmetric MW introduces additional limitations when working in the presence of multi-granularity traffic (i.e., requiring different amount of bandwidth). An example is shown in Fig. 1(d). Two carriers are assumed to be established with a tight channel spacing $B_{1}$. Therefore a connection requiring a bandwidth greater than $B_{1}$ cannot be allocated because any symmetric assignment ( $\left.B_{2}=B_{1}\right)$ overlaps the established connections.

\section{PROPOSED RSTA SCHEME}

This section describes the two proposed RSTA algorithms, specifically, Fig. 3 illustrates the RSTA-N algorithm not supporting slice-ability, whereas Fig. 4 illustrates the RSTA-S algorithm that enables the application of slice-ability.

In the considered dynamic traffic scenario, the slice-ability concept allows to route high bitrate lightpath requests in two possible ways: the first way is to serve the request with a single super-channel; the second way is to serve it by applying sliceability, i.e., with a number of low bitrate lightpaths. The latter solution reduces the spectrum efficiency, but can increase the probability to find a path because routing a number of low bit rate channels can be easier than routing a single high bitrate channel [40], [44]. Thus when slice-ability is enabled, the lightpath requests that cannot be allocated as a single super-channel, exploiting the RSTA-N algorithm, are sliced into $L$ lower bitrate sub-lightpaths to be allocated using the RSTA-S algorithm. Both algorithms exploit the so called slice technology utilization coefficient (STUC) scheme to obtain an ordered list of the available transponder couples, as described in Section III-A.

\section{A. STUC Sorting Scheme}

The STUC scheme has been specifically designed to cope with heterogeneous node equipments, i.e., nodes including both ML-SBVT and MW-SBVT transponders, however, it properly works also when all the transponders installed in the nodes use the same technology. The STUC algorithm selects the most performing available transponder depending on the upcoming lightpath request. The use of MW-SBVTs is favoured when it is able to provide spectrum saving (i.e., at least one frequency slice is saved); this typically happens for lightpath requests to be served with a super-channel, thus allowing to exploit the improved spectral efficiency of MW-SBVT. Otherwise, MLSBVT is used, given that it is able to better accommodated the lightpath requests in the spectrum due to its unconstrained tunability.

The inputs of the sorting scheme are: the bitrate of the incoming lightpath request, and the list of all possible transponder

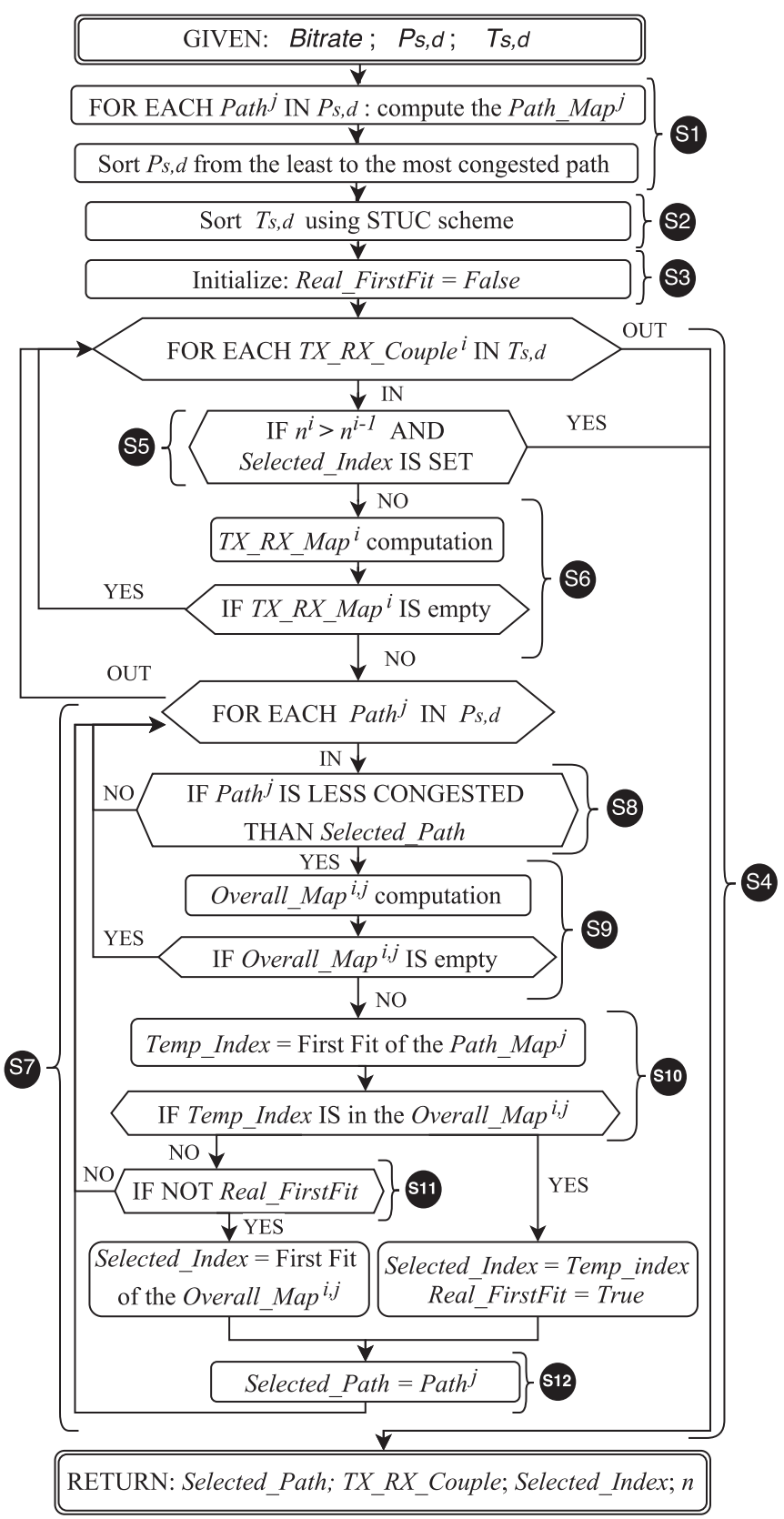

Fig. 3. Flowchart of the proposed RSTA-N scheme.

couples connecting the source node $s$ to the destination node $d$ $\left(T_{s, d}\right)$ having enough free carriers to satisfy the lightpath request. The output is the ordered list of transponder couples in increasing order of number of frequency slices required to serve the considered lightpath request. The required number of frequency slices depends on the technology of the considered transponders couple, if the source and the destination transponders use a different technology, the worst spectrum occupation is considered. Ties are broken considering the transponder technology. Specifically, transponder couples using ML-SBVT technology are preferred with respect to transponder couples using MWSBVT technology. This strategy avoids the utilization of MWSBVT transponders if they do not actually provide spectrum saving. 


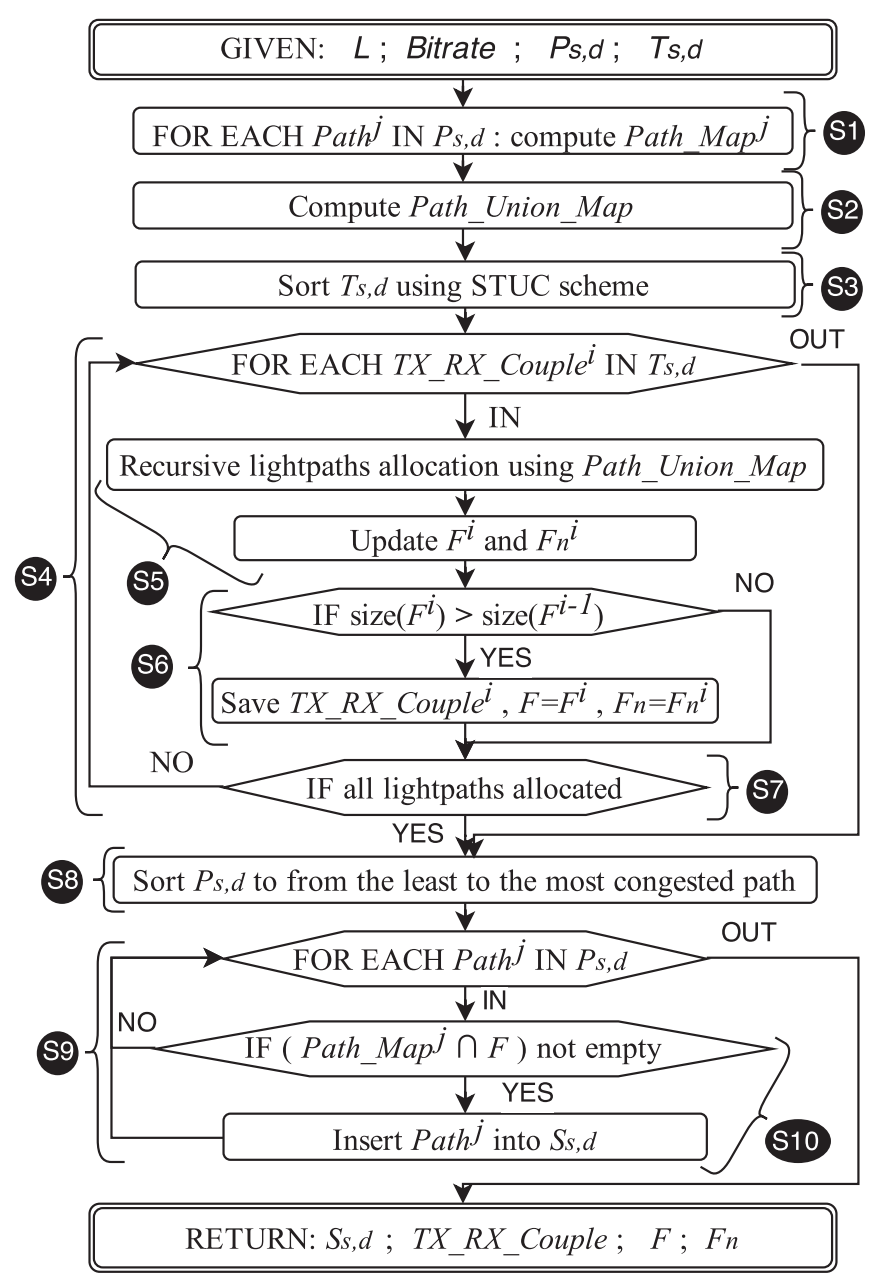

Fig. 4. Flowchart of the proposed RSTA-S scheme. This scheme is used to lightpath requests refused by the RSTA-N scheme, when slice-ability is supported.

Finally, among the transponder couples requiring the same number of frequency slices and using the same technology, the couples with higher utilization coefficient are preferred, i.e., the utilization of already used transponders is favoured. Specifically, the utilization coefficient $\eta$ is computed as follows:

$$
\eta=R_{s} \cdot R_{d}+\epsilon \cdot\left(R_{s}+R_{d}\right)
$$

where $R_{s}$ and $R_{d}$ are defined as the ratio between the number of used carriers and the number of supported carriers by the source and the destination transponder, respectively. $\epsilon$ is a constant small enough to favour the product term with respect to the sum term. In other words, the sum term is only relevant to break ties between transponder couples having equal product term.

\section{B. RSTA-N Scheme}

The RSTA-N algorithm is detailed in Fig. 3. The inputs are: the bitrate to be supported by the incoming lightpath request; the list $P_{s, d}$ of pre-computed and pre-validated candidate paths between the source node $s$ and the destination node $d$; and the list $T_{s, d}$ of all possible transponder couples connecting $s$ to $d$. We assume that the list $P_{s, d}$ includes all the paths from $s$ to $d$ within one hop from the shortest path. The outputs are: a path selected within $P_{s, d}$, a transponder couple (i.e., the transponder indexes to be used at $s$ and $d$ ), the selected central frequency index and the number $n$ of frequency slices to be used for establishing the lightpath request.

Step 1 (see S1 in Fig. 3): The spectrum map $\left(\right.$ Path_Map $\left.^{j}\right)$, i.e., the list of all central frequency indexes available along the path $j$, is computed for each path in $P_{s, d}$. Then, $P_{s, d}$ is sorted from the least congested path to the most congested path. Step 2: The list $T_{s, d}$ is sorted using the STUC scheme. Step 3: Initialize to false a boolean variable (Real_FirstFit) that will be used to indicate whether the first-fit on the path is also supported by the transponder couple. Step 4: The list of transponder couples $T_{s, d}$ is iterated with index $i$, the number of frequency slices required by the considered lightpath request using the $i$ th transponder couple is indicated with $n^{i}$. When all the transponder couples have been considered, the algorithm moves to the final step and returns the outputs. Step 5: If $n^{i}$ is higher than $n^{i-1}$ and a potential frequency index (Selected_Index) has been already selected, the algorithm jumps to the final step and returns the outputs. This step breaks the loop when all remaining transponder pairs in the list require a higher number of frequency slices to serve the lightpath. Step 6: The spectrum map of the $i$ th transponder couple ( $T X_{-} R X_{-} M a p^{i}$ ) is computed, i.e., the list of all available central frequency indexes that can be used to accommodate the lightpath. If $T X_{-} R X_{-} M a p^{i}$ is empty, the next transponder couple is selected and the algorithm moves back to the beginning of step 4. Step 7: The list of paths $P_{s, d}$, ordered at step 1, is iterated using index $j$. Step 8: If the path under consideration $\left(P a t h^{j}\right)$ is less congested than a path selected in a previous iteration (Selected_Path), the algorithm continues to Step 9, otherwise it jumps back to step 7 to consider the next path. Step 9: The spectrum map of the $j$ th path, Path_Map ${ }^{j}$, is computed (i.e., the list of all central frequency indexes available along the path). The Path_Map ${ }^{j}$ is intersected with $T X_{-} R X_{-} M a p^{i}$ to obtain the Overall_Map ${ }^{i, j}$ containing all the central frequency indexes supported by transponder couple $i$ and path $j$. If Overall_Map ${ }^{i, j}$ is empty, the algorithm moves back to the beginning of Step 7. Step 10: First-fit spectrum assignment is performed on the Path_Map ${ }^{j}$, i.e., the first frequency index available in the path is selected and saved to a temporary variable (Temp_Index). If Temp_Index is also available in the Overall_Map ${ }^{i, j}$, i.e., it is supported by the transponder couple, it is saved as Selected_index, the Real_FirstFit variable is set to True, and the algorithm continues to Step 12. Otherwise, the algorithm moves to the next step. Step 11: The transponder couple under consideration does not support the Temp_Index. If the saved Selected_Index satisfies the Real_FirstFit (Real_FirstFit is True), i.e., if in a previous iteration the Temp_Index was supported by the transponder couple, the algorithm jumps back to step 7 without overwriting the Selected_Index. Otherwise, the Selected_Index is overwritten by applying the first-fit on the Overall_Map ${ }^{i, j}$. Step 12: The Path ${ }^{j}$ is saved as Selected_Path. The algorithm moves back to step 7. If no resources are found to establish the lightpath requests the algorithm fails and the lightpath request is refused. 
The described scheme first favours the utilization of a transponder couple using the minimum number of frequency slices for establishing the considered lightpath request. Second, it favours the utilization of a path in which a first-fit spectrum assignment, made on the list of indexes available in the path (Path_Map), is also supported by the associated transponder couple. Third, it favours the use of the least congested path. The former target aims at maximizing the spectrum efficiency, the second target aims at reducing the spectrum fragmentation, finally the third target aims at increasing the load balancing. The implemented RSTA-N scheme considers asymmetrical multiwavelength sources, however it could be easily adapted to symmetrical multi-wavelength sources by properly considering the additional spectrum constraint during the computation of the $T X_{-} R X_{-} M a p$.

\section{RSTA-S Scheme}

When slice-ability is enabled, for those lightpath requests that have been refused by the RSTA-N algorithm, the RSTA-S algorithm is performed as detailed in Fig. 4. Specifically, the lightpath requests that cannot be allocated using a single superchannel are sliced into $L$ lower bitrate sub-lightpaths. The generated sub-lightpaths do not require to be allocated contiguously in the spectrum, , and we assume that they can be routed along different paths (i.e., multi-path slice-ability). In this way sliceability provides the maximum benefit to the lightpath blocking probability [36], [37]. However, according with the considered SBVT architecture [12], all the sub-lightpaths must be served by the same SBVT at both source and destination nodes. Therefore, $L \leq N$ where $N$ is the number of optical sub-carriers generated by the considered SBVT.

The inputs of the RSTA-S algorithm are: the number $L$ of sub-lightpaths to be allocated; the bitrate of each lightpath; the list $P_{s, d}$ of pre-computed paths; and the list $T_{s, d}$ of all possible transponder couples. The outputs are: a list of paths $S_{s, d}$ selected within $P_{s, d}$, one path for each successfully routed sub-lightpath; a single transponder couple; and, a list $F$ of the central frequency indexes, one per lightpath, with the corresponding list of the number of required frequency slices $F_{n}$.

Step 1: For each path $j$ in $P_{s, d}$, compute the spectrum map $\left(P_{a t h} \mathrm{Map}^{j}\right)$. Step 2: Compute Path_Union_Map given by the union the $P a t h_{-} M a p^{j}$ for all the paths in $P_{s, d}$, i.e., a list containing all central frequency indexes which are available along at least one path in $P_{s, d}$. Step 3: The list $T_{s, d}$ is sorted using the STUC sorting scheme. Step 4: The list of transponder couples $T_{s, d}$ is iterated with index $i$. Step 5: Recursively allocate the sub-lightpaths on the Path_Union_Map using first-fit spectrum assignment while saving the used central frequency indexes in $F$ and the corresponding number of frequency slices in $F_{n}$. Step 6: Keep track of the transponder couple enabling the allocation of the highest number of sub-lightpaths. Step 7: In case the transponder couple $i$ is able to allocate all the $L$ sub-lightpaths, the algorithm breaks the iteration and continues to step 8. Step 8: Sort $P_{s, d}$ from the least to the most congested path. Step 9: The ordered list of paths $P_{s, d}$ is iterated using index $j$. Step 10: Check whether the path $j$ support at least one

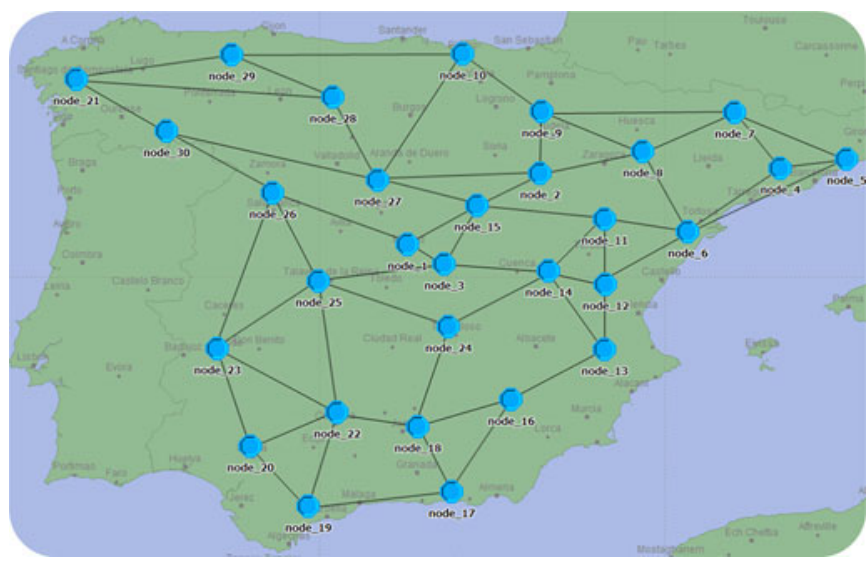

Fig. 5. Spanish backbone network topology.

central frequency index contained in $F$, i.e., if the intersection of $P a t h \_M a p^{j}$ with $F$ is not empty. If not empty, add the path to $S_{s, d}$. Final Step: Return the outputs.

\section{Performance Evaluation}

\section{A. Control Plane Scenario}

The proposed algorithms are evaluated considering a distributed control plane based on Generalized Multi-Protocol Label Switching with a centralized Path Computation Element (i.e., GMPLS/PCE control plane) [12]. Specifically, a stateful PCE is considered to store a Traffic Engineering Database (TED) and a Label Switched Path (i.e., lightpath) Database (LSP-DB) [45]. The TED stores the network topology, spectrum availability and transponder state information, updated through both the Open Shortest Path First routing protocol with Traffic Engineering extensions (i.e., OSPF-TE) and direct PCE Protocol (PCEP) messages [46]. The LSP-DB includes information about all the lightpaths currently established in the network and it is kept up to date through PCEP messages. The additional transponder state information introduced in the TED are used at the PCE during the execution of the proposed RSTA algorithms.

When a lightpath request is generated, the source node sends a PCEP path computation request to the PCE asking for a path. The PCE performs the proposed RSTA and replies with a PCRep message to the requesting node including the path, the TX/RX transponders the central frequency and the number of frequency slices to be used. Finally, the source node triggers the Resource Reservation Protocol with Traffic Engineering extensions (RSVP-TE) to actually establish the lightpath.

\section{B. Simulation Scenario}

Simulations are performed using OPNET Modeler [47]. The developed model includes an implementation of the RSVP-TE, OSPF-TE and PCEP protocols with the extensions required for EONs supporting different transponder technologies.

The test network is represented in Fig. 5 that consists of $\mathcal{V}=30$ nodes and $\mathcal{E}=56$ bidirectional links with $\mathcal{F}=256$ frequency slices per direction, thus considering a total spectrum 
of 3.2 THz. The PCE is located at node 1, and for each lightpath request from node $s$ to node $d$ the set of candidate paths $P_{s, d}$ includes all the paths within one hop from the shortest path.

The considered transponders are capable of generating up to four optical carriers $(N=4)$, thus each transponder is equipped with four modulators and one laser (MW-SBVT technology) or four lasers (ML-SBVT technology). In the MW-SBVT case the maximum spacing allowed between carriers is $50 \mathrm{GHz}$ as explained in Section II. $\mathcal{R}=2$ bitrate values are considered for lightpath requests: $100 \mathrm{~Gb} / \mathrm{s}$ lightpaths use one optical carrier occupying three slices $(37.5 \mathrm{GHz}) ; 400 \mathrm{~Gb} / \mathrm{s}$ lightpaths use four optical carriers occupying nine slices $(112.5 \mathrm{GHz})$ in case of ML-SBVT or eight slices $(100 \mathrm{GHz})$ in case MW-SBVT [39].

Two traffic profiles are analysed according to the considered lightpath bitrates. In both scenarios the traffic matrix is uniformly distributed among node pairs, lightpath requests arrive following a Poisson process, and the average lightpath service time is fixed to $1 \mathrm{~h}$. In the $400 \mathrm{G}$ traffic profile only $400 \mathrm{~Gb} / \mathrm{s}$ lightpath requests are considered. In the HYBRID traffic profile both 100 and $400 \mathrm{~Gb} / \mathrm{s}$ lightpath requests are considered. The network load offered to the network is computed according to Eq. (2), and in the HYBRID scenario it is equally composed of 100 and $400 \mathrm{~Gb} / \mathrm{s}$ lightpath requests.

$$
\Lambda=\sum_{v=1}^{\mathcal{V}} \sum_{r=1}^{\mathcal{R}} \frac{\lambda_{v, r}}{\mu_{v, r}} \cdot \frac{r}{r_{\min }}
$$

where $v$ is the node index, $r$ is the demand bitrate (e.g., 100 or $400 \mathrm{~Gb} / \mathrm{s}), 1 / \lambda_{v, r}$ is the mean inter-arrival time for lightpath requests of rate $r$ generated by node $v, 1 / \mu_{v, r}$ is the mean service time for lightpath requests of rate $r$ generated by node $v$, and $r_{\min }$ is a weight factor taking into account the lightpath request bitrate with respect to the minimum allowed bitrate (i.e., $r_{\min }=100 \mathrm{~Gb} / \mathrm{s}$ ).

For each traffic profile, three node architectures are compared: MW node architecture (100\%MW), ML node architecture $(100 \% \mathrm{ML})$ and heterogeneous node architecture $(x \% \mathrm{MW}-$ $y \% \mathrm{ML})$. In MW and ML architectures, all network nodes are fully equipped with a single transponder technology, i.e., MWSBVTs or ML-SBVTs, respectively. In the heterogeneous architecture, the network nodes are equipped with both MW-SBVT and ML-SBVT technologies in a variable percentage, i.e., $x$ and $y$, respectively. For each node architecture, two cases are considered depending on the capability of the transponders to support slice-ability.

The considered scenarios are evaluated in terms of blocking probability $\left(P_{b}\right) . P_{b}$ is defined as the ratio between the blocked bandwidth expressed in bitrate and the overall requested bandwidth. In order to better characterize the different transponder technologies, $P_{b}$ is studied as a function of the number of transponders installed on each node when the network load is fixed. Then, as a function of the network load with a fixed number of transponders per node.

All scenarios consider a total simulation time of 500 days for each simulated point.

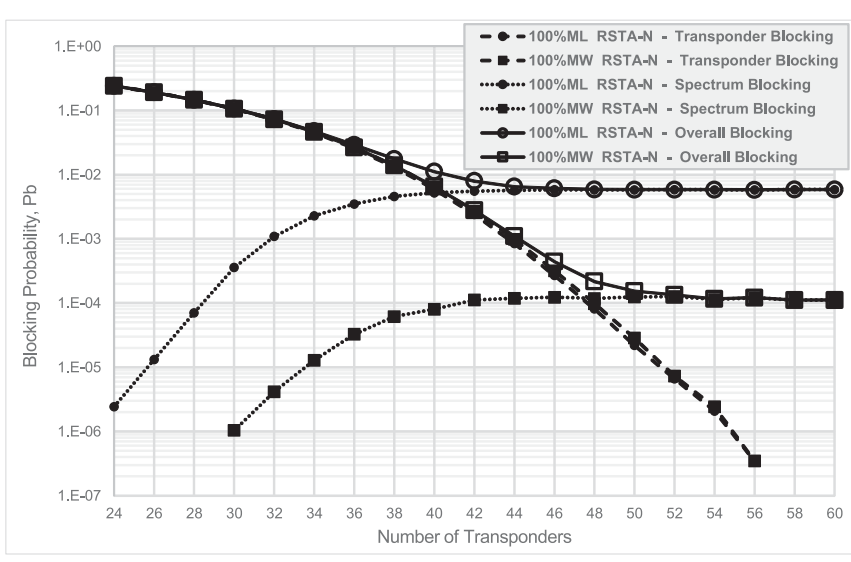

Fig. 6. RSTA-N scheme in the $400 \mathrm{G}$ traffic profile, $P_{b}$ as function of number of transponders per node. Network load is fixed to 1600 Erlang.

\section{Simulation Results}

1) $400 G$ Traffic Profile: Fig. 6 shows $P_{b}$ as a function of the number of transponders per node when RSTA-N scheme is applied and the network has a fixed load of 1600 Erlang. Specifically, the figure compares the overall blocking probability for the ML and the MW node architectures to quantify the benefits provided by MW-SBVT when high bitrate super-channels are considered. The overall blocking probability is formed by two contributions: the transponder blocking and the spectrum blocking. The transponder blocking contribution counts for the lightpaths blocked due to lack of transponders, whereas the spectrum blocking contribution counts for the lightpaths blocked due to spectrum unavailability (i.e., continuity constraint, contiguity constraint, or spectrum lack [12]). Results show that ML and MW node architectures experience similar blocking due to the lack of transponders, but the MW node architecture performs much better in terms of spectrum blocking. Therefore, the spectrum compression introduced by the multi-wavelength, i.e., eight frequency slices are used instead of nine to establish a 400 $\mathrm{Gb} / \mathrm{s}$ lightpath, provides important benefit to the network, in particular when the number of transponders per node is sufficient to sustain the network load. Indeed, the blocking probability is reduced of almost two orders of magnitude using a MW-SBVT technology.

Fig. 7 shows $P_{b}$ as a function of the network load when the number of transponders per node is fixed to 45 . Results demonstrate that the achieved improvements introduced with the higher spectrum efficiency of the MW-SBVT are valid for a wide range of loads.

2) HYBRID Traffic Profile: Fig. 8 shows $P_{b}$ as a function of the number of transponders per node with fixed network load of 1000 Erlang. Besides MW and ML node architectures, the heterogeneous node architecture is included in this analysis. For each architecture the performance of the proposed RSTA-N scheme is compared with an RSA scheme performing the same steps of the proposed RSTA-N scheme but without knowledge of the transponders status and technologies. Using this RSA scheme the PCE does not select a transponders pair that is 


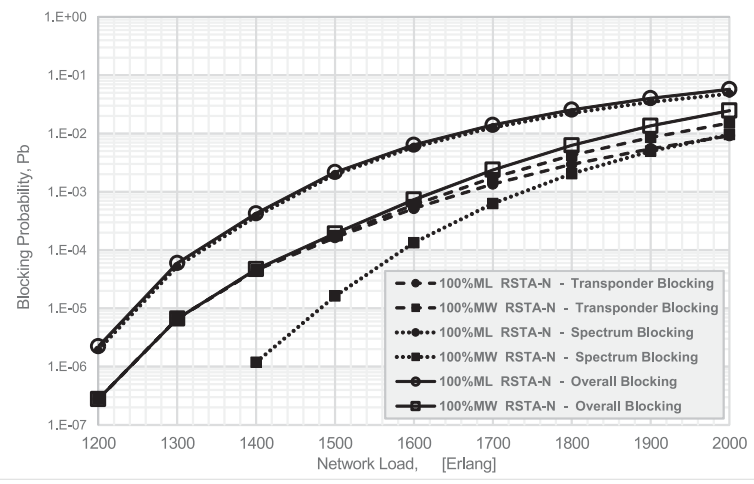

Fig. 7. RSTA-N scheme in the $400 \mathrm{G}$ traffic profile, $P_{b}$ as function of network load. 45 transponders per node are considered.

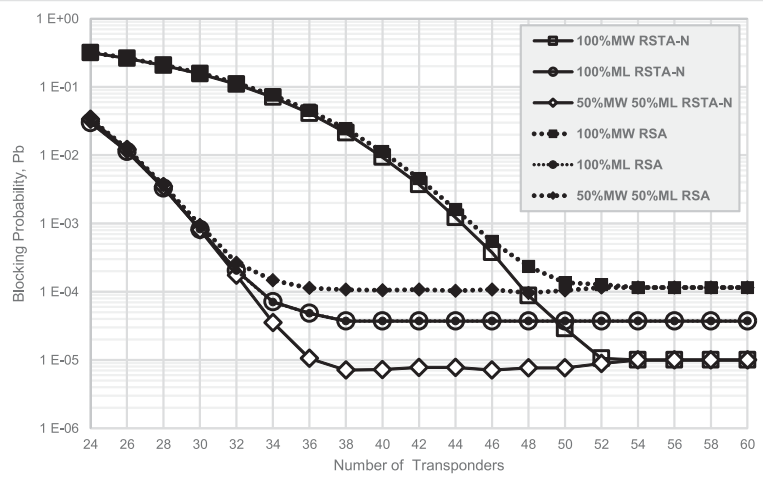

Fig. 8. RSTA-N scheme versus RSA in the HYBRID traffic profile, $P_{b}$ as function of number of transponders per node. Network load is fixed 1000 Erlang.

instead locally assigned during the distributed signaling procedure. Fig. 8 shows that with the ML node architecture RSTA-N does not provide benefit with respect to traditional RSA, indeed ML-SBVTs do not introduce specific constraints, and this is the reason why RSTA algorithms had not been proposed so far. Conversely, in both cases using MW-SBVTs the proposed RSTA-N scheme guarantees a significant blocking probability improvement with respect to the traditional RSA since the using MW-SBVTs introduce specific constraints that have to be taken into account during the computation phase. It is interesting to notice that the heterogeneous node architecture achieves the best performance when RSTA-N is applied, while if traditional RSA is applied the presence of the two technologies degrades the network performance with respect to the utilization of ML-SBVT only.

Fig. 9 compares RSTA-N and RSA algorithms in terms of $P_{b}$ as a function of the offered network load. The number of transponders per node is fixed to 50. Results show that the $P_{b}$ improvement obtained with RSTA-N is significant for MW and hybrid node architectures. In particular, the hybrid node architecture benefits most from RSTA-N achieving the lowest $P_{b}$ followed by the MW node architecture. As expected, similarly to Fig. 8 the $P_{b}$ remains the same for both algorithms with the ML node architecture.

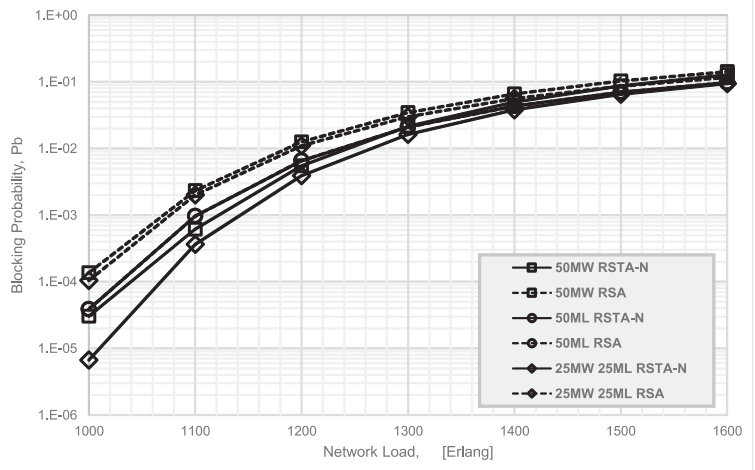

Fig. 9. $\quad$ RSTA-N scheme versus RSA in the HYBRID traffic profile, $P_{b}$ as function of the traffic load. The number of transponders is fixed to 50 .

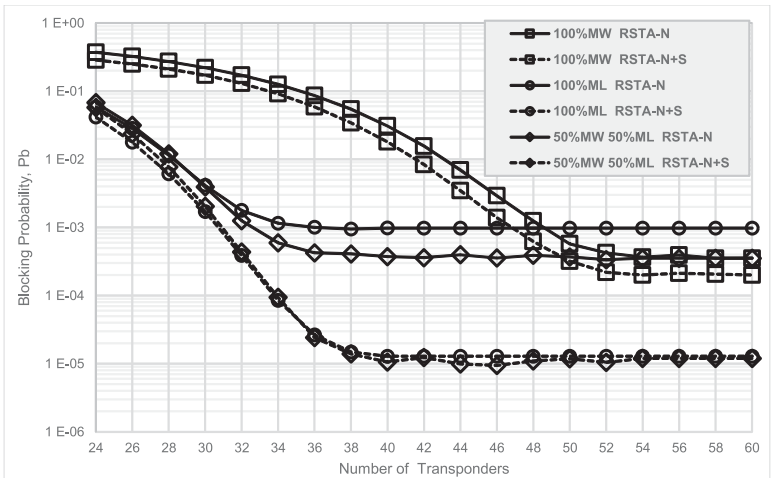

Fig. 10. Slice-ability assessment, RSTA-N scheme versus RSTA-N+S scheme in the HYBRID traffic profile; $P_{b}$ as function of number of transponders per node. Network load is fixed 1100 Erlang.

Fig. 10 shows $P_{b}$ as a function of the number of transponders per node with fixed network load of 1100 Erlang. Solid lines shows the achieved performance by the RSTA-N scheme that does not use slice-ability, whereas dashed lines consider the application of slice-ability where RSTA-N and RSTA-S are applied sequentially as explained in Section III (i.e., see RSTA$\mathrm{N}+\mathrm{S}$ in Fig. 10). The results illustrated in Fig. 10 demonstrates that the ML node architecture achieves better results with respect to the MW node architecture when $P_{b}$ is dominated by the lack of transponders. Increasing the number of transponders per node, $P_{b}$ is dominated by the spectrum blocking and the situation is reversed, i.e., the MW node architecture outperforms the ML node architecture thanks to the higher spectrum efficiency.

The most important result in Fig. 10 is about the achievable performance using the heterogeneous node architecture. In this case, where each node is equipped with $50 \% \mathrm{MW}-\mathrm{SBVT}$ and 50\% ML-SBVT, the proposed RSTA-N algorithm allows to benefit from both the transponder technologies. Specifically, the heterogeneous architecture performs similarly to the ML architecture for very limited number of transponders per node (i.e., lower than 30), while it performs similarly to the MW architecture for high number of transponders per node (i.e., higher than 52), in other words the heterogeneous architecture 


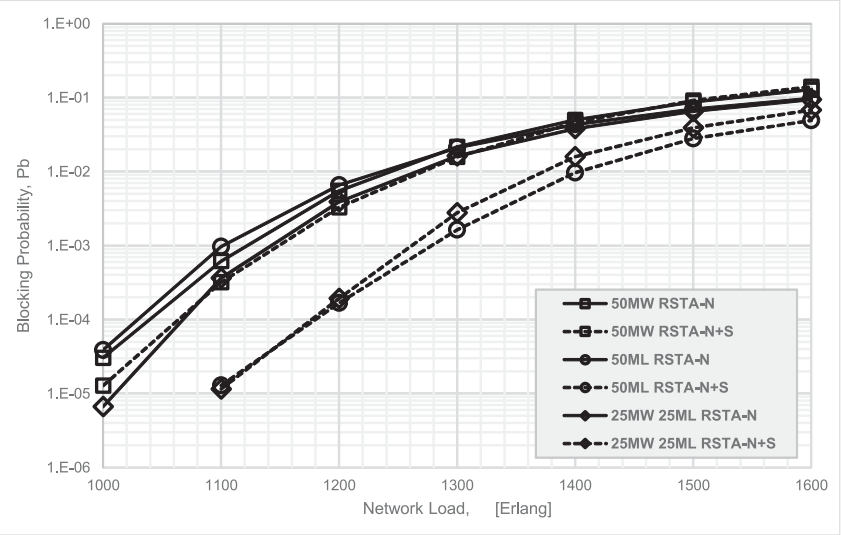

Fig. 11. Slice-ability assessment, RSTA-N scheme versus RSTA-N+S scheme in the HYBRID traffic profile; $P_{b}$ as function of the network load. The number of transponders is fixed to 50 .

exploits the benefits of both technology architectures. Moreover, the heterogeneous node architecture significantly outperforms both the single technology architectures achieving lowest $P_{b}$.

When the slice-ability is applied (see RSTA-N+S curves in Fig. 10), the $400 \mathrm{~Gb} / \mathrm{s}$ lightpath requests that are refused by the RSTA-N scheme uses the RSTA-S scheme with $L=4$ exploiting slice-ability to establish four $100 \mathrm{~Gb} / \mathrm{s}$ sub-lightpaths. In this case, the most relevant improvement is achieved by the ML architecture for which the $P_{b}$ decreases of two orders of magnitude approximately. Conversely, the MW node architecture only slightly benefits from slice-ability due to the inherent constraint of MW-SBVTs sub-carrier tunability, i.e., the maximum spacing among the generated sub-carriers is $50 \mathrm{GHz}$, see Section II. Finally, when slice-ability is applied to the heterogeneous node architecture, a very low $P_{b}$ is also achieved comparable to the case of ML node architecture.

Fig. 11 compares RSTA-N and RSTA-N+S algorithms in terms of $P_{b}$ as a function of the offered network load. The number of transponders per node is fixed to 50. Results show that the improvement offered by the slice-ability is present also varying the network load. In particular, the $P_{b}$ gain achieved with slice-ability is more pronounced at lower network load and decreases as the load increases. However, it is important to underline that $P_{b}$ above $10^{-2}$ is in general not tolerated in real networks. Similarly to Fig. 10, ML and hybrid node architectures achieve the lowest $P_{b}$ with slice-ability. For values of network load lower than 1300 Erlang $\left(P_{b}\right.$ lower than $\left.10^{-2}\right)$, the gain achieved through the slice-ability is more than one order of magnitude for ML and hybrid node architectures and half order of magnitude for MW node architecture.

Fig. 12 considers three hybrid traffic profiles with increasing percentage of $400 \mathrm{~Gb} / \mathrm{s}$ traffic $(40 \%, 60 \%, 80 \%)$ and reports the performance of the proposed RSTA with respect to the traditional RSA scheme. The network load is fixed to 1100 Erlang. Results show that the benefits of the proposed RSTA scheme increase if a higher percentage of $400 \mathrm{~Gb} / \mathrm{s}$ request is present. Specifically, focusing at the point with 50 transponders per node the blocking probability improvement is $78 \%$ with $40 \%$ of 400

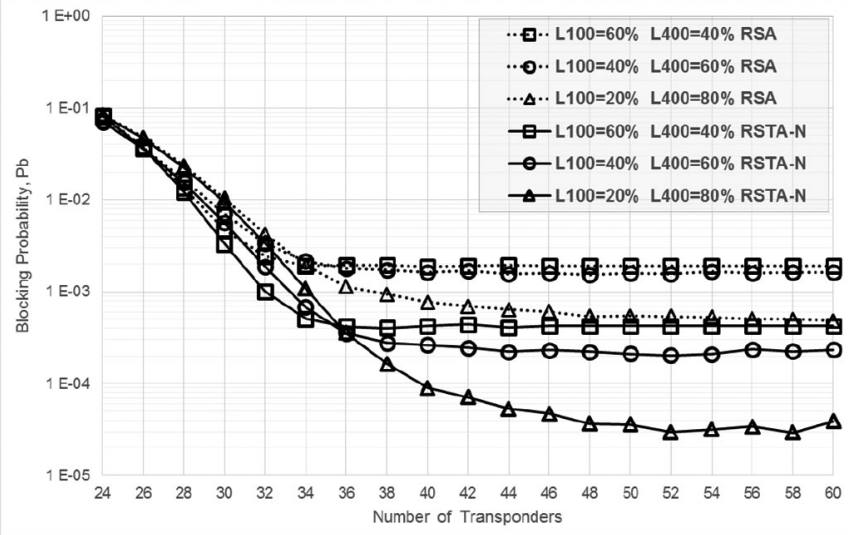

Fig. 12. RSTA-N scheme vs RSA in various HYBRID traffic profiles, $P_{b}$ as function of number of transponders per node. Network load is fixed 1100 Erlang.

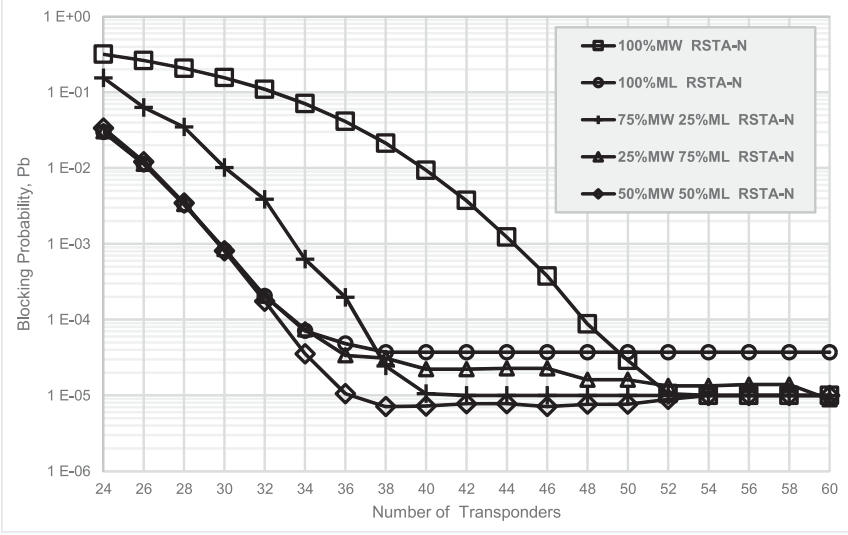

Fig. 13. Influence of node transponder equipment with RSTA-N scheme, $P_{b}$ as function of number of transponders per node. Network load is 1000 Erlang.

$\mathrm{Gb} / \mathrm{s}$ requests, $87 \%$ with $60 \%$ of $400 \mathrm{~Gb} / \mathrm{s}$ requests and $94 \%$ with $80 \%$ of $400 \mathrm{~Gb} / \mathrm{s}$ requests.

3) Influence of Node Transponder Equipment: In this section the heterogeneous node architecture is evaluated with different percentage of ML-SBVTs and MW-SBVTs, the HYBRID traffic profile is used. Three different node transponder equipments are considered, i.e., $75 \% \mathrm{MW}-25 \% \mathrm{ML}, 50 \% \mathrm{MW}-$ $50 \% \mathrm{ML}$, and 25\%MW-75\%ML.

Fig. 13 shows $P_{b}$ as a function of number of transponders per node and for different heterogeneous node architectures when slice-ability is not applied (RSTA-N scheme), single technology node architectures curves are included for comparison. Results show that among all considered node transponder equipments, the 50\%MW-50\%ML achieves the best $P_{b}$. This is due to the considered HYBRID traffic profile in which $50 \%$ of the network load given by $400 \mathrm{~Gb} / \mathrm{s}$ lightpath requests and the other $50 \%$ given by $100 \mathrm{~Gb} / \mathrm{s}$ lightpath requests. Indeed, network resources are used in the optimal way if all the $400 \mathrm{~Gb} / \mathrm{s}$ lightpath requests are served by MW-SBVTs and all the $100 \mathrm{~Gb} / \mathrm{s}$ lightpath requests are served by ML-SBVTs, thus a proper balancing is required between the offered traffic profile and the node 


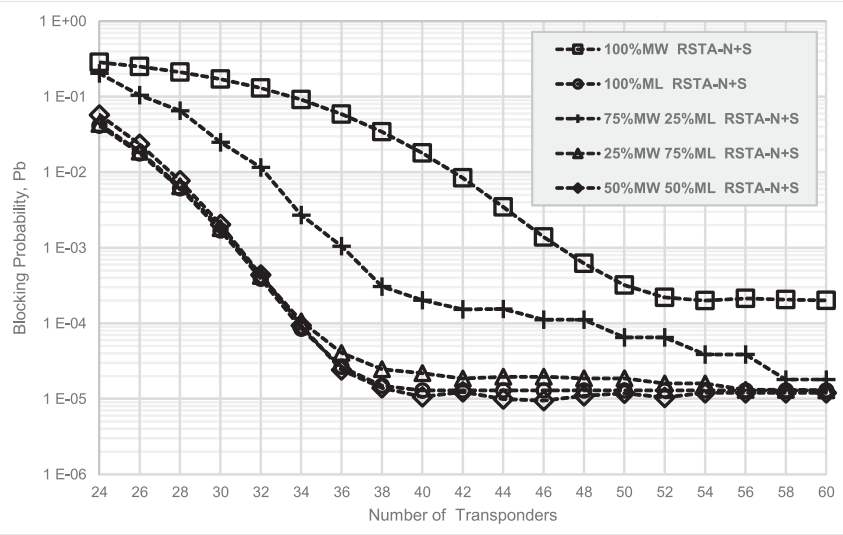

Fig. 14. Influence of node transponder equipment with RSTA-N+S scheme, $P_{b}$ as function of number of transponders per node. Network load is 1100 Erlang.

transponder equipment. Specifically, for a low number of transponders per node, $75 \% \mathrm{MW}-25 \% \mathrm{ML}$ experiences worse $P_{b}$ being in between the $100 \% \mathrm{MW}$ curve and the $100 \% \mathrm{ML}$ curve; whereas the $25 \% \mathrm{MW}-75 \% \mathrm{ML}$ curve lies on the $100 \% \mathrm{ML}$ curve. This happens because with few transponders per node $P_{b}$ is dominated by the lack of transponders, thus it is beneficial to have more transponders with higher flexibility (i.e. ML-SBVTs). Increasing the number of transponders per node, $75 \% \mathrm{MW}-$ $25 \% \mathrm{ML}$ rapidly approach the $50 \% \mathrm{MW}-50 \% \mathrm{ML}$ curve, while the $25 \% \mathrm{MW}-75 \% \mathrm{ML}$ curve needs a higher number of transponders per node to converge. This is because with many transponders per node $P_{b}$ is dominated by the lack of spectrum, thus it is beneficial to have more transponders with higher spectrum efficiency (i.e. MW-SBVTs).

In Fig. 14 slice-ability is applied using the RSTA-N+S scheme. When few transponders per node are available, the general behaviour is similar to the case without slice-ability. For higher number of transponders per node, having more MLSBVTs transponders (even 100\%ML and 25\%MW-75\%ML) provides $P_{b}$ similar to the $50 \% \mathrm{MW}-50 \% \mathrm{ML}$ case, whereas the $75 \% \mathrm{MW}-25 \% \mathrm{ML}$ needs a higher number of transponders per node to converge, because it essentially converges when the number of MW-SBVTs per node is enough to effectively apply slice-ability.

\section{CONCLUSION}

In the EON architecture SBVTs use multiple optical carriers to support the generation of high bitrate super-channels enabling increased spectral efficiency. SBVTs typically use a dedicated laser to generate each carrier (ML-SBVT) or a single laser source to generate multiple optical carriers (MW-SBVT). In the latter case the super-channel spectrum efficiency is considerably improved, but new constraints to the RSA are introduced because of limited tunability of generated optical carriers.

Taking into account the introduced constraints this paper integrated the selection of the transponder with the RSA thus proposing a dynamic RSTA scheme supporting both ML-SBVT and MW-SBVT technologies. The proposed scheme is able to combine the benefits of the two technologies. Specifically, simulation results showed that the proposed RSTA schemes provide benefit with respect to traditional RSA in terms of achieved blocking probability and demonstrated that the best network design solution is to deploy heterogeneous nodes equipped with transponders based on both SBVT technologies. Simulations also revealed that, when all the traffic is composed by high bitrate super-channels, MW-SBVT is a very attractive technology.

\section{REFERENCES}

[1] M. Dallaglio, A. Giorgetti, N. Sambo, L. Velasco, and P. Castoldi, "Impact of multi-wavelength sliceable transponders in elastic optical networks," presented at the Tech. Dig. Optical Fiber Communication Conf., Los Angeles, CA, USA, Mar. 2015.

[2] M. Jinno, H. Takara, Y. Sone, K. Yonenaga, and A. Hirano, "Multiflow optical transponder for efficient multilayer optical networking," IEEE Commun. Mag., vol. 50, no. 5, pp. 56-65, May 2012.

[3] O. Gerstel, M. Jinno, A. Lord, and S. Yoo, "Elastic optical networking: A new dawn for the optical layer?" IEEE Commun. Mag., vol. 50, no. 2, pp. s12-s20, Feb. 2012.

[4] M. Jinno, B. Kozicki, H. Takara, A. Watanabe, Y. Sone, T. Tanaka, and A. Hirano, "Distance-adaptive spectrum resource allocation in spectrumsliced elastic optical path network," IEEE Commun. Mag., vol. 48, no. 8, pp. 138-145, Aug. 2010.

[5] T. Ohara, M. Teshima, S. Aisawa, and M. Jinno, "OTN technology for multi-flow optical transponder in elastic 400G/1T transmission era," presented at the Optical Fiber Communication Conf. Expo., Nat. Fiber Optic Engineers Conf., Los Angeles, CA, USA, 2012.

[6] N. Sambo, A. D’Errico, C. Porzi, V. Vercesi, M. Imran, F. Cugini, A. Bogoni, L. Potì, and P. Castoldi, "Sliceable transponder architecture including multiwavelength source," J. Opt. Commun. Netw., vol. 6, no. 7, pp. 590-600, Jul. 2014.

[7] "Interfaces for the optical transport network," Recommendation G.709/Y.1331, Feb. 2012.

[8] N. Sambo, G. Meloni, F. Paolucci, M. Imran, F. Fresi, F. Cugini, P. Castoldi, and L. Poti, "First demonstration of SDN-controlled SBVT based on multiwavelength source with programmable and asymmetric channel spacing," presented at the European Conf. Optical Communication, Cannes, France, Sep. 2014.

[9] V. Lopez, B. de la Cruz, O. Gonzalez de Dios, O. Gerstel, N. Amaya, G. Zervas, D. Simeonidou, and J. Fernandez-Palacios, "Finding the target cost for sliceable bandwidth variable transponders," J. Opt. Commun. Netw., vol. 6, no. 5, pp. 476-485, May 2014.

[10] B. de la Cruz, O. Gonzalez de Dios, V. Lopez, and J. Fernandez-Palacios, "Opex savings by reduction of stock of spare parts with sliceable bandwidth variable transponders," presented at the Optical Fiber Communication Conf. Exhib., San Francisco, CA, USA, Mar. 2014.

[11] J. Zhang, Y. Zhao, X. Yu, J. Zhang, M. Song, Y. Ji, and B. Mukherjee, "Energy-efficient traffic grooming in sliceable-transponder-equipped IPover-elastic optical networks [invited]," J. Opt. Commun. Netw., vol. 7, no. 1, pp. A142-A152, Jan. 2015.

[12] M. Dallaglio, A. Giorgetti, N. Sambo, F. Cugini, and P. Castoldi, "Provisioning and restoration with sliceability in GMPLS-based elastic optical networks [invited]," J. Opt. Commun. Netw., vol. 7, no. 2, pp. A309-A317, Feb. 2015.

[13] N. Sambo, P. Castoldi, A. D’Errico, E. Riccardi, A. Pagano, M. S. Moreolo, J. M. Fabrega, D. Rafique, A. Napoli, S. Frigerio, E. H. Salas, G. Zervas, M. Nolle, J. K. Fischer, A. Lord, and J. Gimenez, "Next generation sliceable bandwidth variable transponders," IEEE Commun. Mag., vol. 53, no. 2, pp. 163-171, Feb. 2015.

[14] A. Napoli, M. Bohn, D. Rafique, A. Stavdas, N. Sambo, L. Poti, M. Nolle, J. K. Fischer, E. Riccardi, A. Pagano, A. Di Giglio, M. S. Moreolo, J. M. Fabrega, E. Hugues-Salas, G. Zervas, D. Simeonidou, P. Layec, A. D'Errico, T. Rahman, and J. P. F.-P. Gimenez, "Next generation elastic optical networks: The vision of the european research project IDEALIST," IEEE Commun. Mag., vol. 53, no. 2, pp. 152-162, Feb. 2015.

[15] H. Takara, T. Goh, K. Shibahara, K. Yonenaga, S. Kawai, and M. Jinno, "Experimental demonstration of $400 \mathrm{~Gb} / \mathrm{s}$ multi-flow, multi-rate, multireach optical transmitter for efficient elastic spectral routing," presented at the European Conf. Exhib. Optical Communication, Geneva, Switzerland, Sep. 2011 
[16] J. Fabrega, M. Svaluto Moreolo, F. Vflchez, B. Rofoee, Y. Ou, N. Amaya, G. Zervas, D. Simeonidou, Y. Yoshida, and K. Kitayama, "Experimental demonstration of elastic optical networking utilizing time-sliceable bitrate variable OFDM transceiver," presented at the Optical Fiber Communication Conf. Exhib., San Francisco, CA, USA, Mar. 2014.

[17] P. Anandarajah, R. Zhou, R. Maher, M. Gutierrez Pascual, F. Smyth, V. Vujicic, and L. Barry, "Flexible optical comb source for super channel systems," presented at the Optical Fiber Communication Conf. Expo. Nat. Fiber Optic Engineers Conf., Anaheim, CA, USA, Mar. 2013.

[18] M. Klinkowski and K. Walkowiak, "Routing and spectrum assignment in spectrum sliced elastic optical path network," IEEE Commun. Lett., vol. 15, no. 8, pp. 884-886, Aug. 2011.

[19] L. Velasco, A. Castro, M. Ruiz, and G. Junyent, "Solving routing and spectrum allocation related optimization problems: From off-line to inoperation flexgrid network planning," J. Lightw. Technol., vol. 32, no. 16 , pp. 2780-2795, Aug. 2014.

[20] W. Shi, Z. Zhu, M. Zhang, and N. Ansari, "On the effect of bandwidth fragmentation on blocking probability in elastic optical networks," IEEE Trans. Commun., vol. 61, no. 7, pp. 2970-2978, Jul. 2013.

[21] M. Tornatore, C. Rottondi, R. Goscien, K. Walkowiak, G. Rizzelli, and A. Morea, "On the complexity of routing and spectrum assignment in flexible-grid ring networks [invited]," J. Opt. Commun. Netw., vol. 7 , no. 2, pp. A256-A267, Feb. 2015.

[22] K. Christodoulopoulos, I. Tomkos, and E. Varvarigos, "Elastic bandwidth allocation in flexible OFDM-based optical networks," J. Lightw. Technol., vol. 29, no. 9, pp. 1354-1366, May 2011.

[23] Y. Wang, X. Cao, Q. Hu, and Y. Pan, "Towards elastic and fine-granular bandwidth allocation in spectrum-sliced optical networks," J. Opt. Commun. Netw., vol. 4, no. 11, pp. 906-917, Nov. 2012.

[24] A. Cai, G. Shen, L. Peng, and M. Zukerman, "Novel node-arc model and multiiteration heuristics for static routing and spectrum assignment in elastic optical networks," J. Lightw. Technol., vol. 31, no. 21, pp. 3402 3413, Nov. 2013.

[25] S. Talebi, E. Bampis, G. Lucarelli, I. Katib, and G. Rouskas, "Spectrum assignment in optical networks: A multiprocessor scheduling perspective," J. Opt. Commun. Netw., vol. 6, no. 8, pp. 754-763, Aug. 2014.

[26] N. Sambo, F. Cugini, G. Bottari, G. Bruno, P. Iovanna, and P. Castoldi, "Lightpath provisioning in wavelength switched optical networks with flexible grid," presented at the European Conf. Exhib. Optical Communication, Geneva, Switzerland, Sep. 2011.

[27] X. Wan, N. Hua, and X. Zheng, "Dynamic routing and spectrum assignment in spectrum-flexible transparent optical networks," J. Opt. Commun. Netw., vol. 4, no. 8, pp. 603-613, Aug. 2012.

[28] H. Beyranvand and J. Salehi, "A quality-of-transmission aware dynamic routing and spectrum assignment scheme for future elastic optical networks," J. Lightw. Technol., vol. 31, no. 18, pp. 3043-3054, Sep. 2013.

[29] N. Sambo, G. Meloni, F. Cugini, A. D'Errico, L. Potì, P. Iovanna, and P. Castoldi, "Routing, code, and spectrum assignment (RCSA) in elastic optical networks," presented at the Optical Fiber Communication Conf. Expo., Nat. Fiber Optic Engineers Conf., Los Angeles, CA, USA, Mar. 2015.

[30] A. Rosa, C. Cavdar, S. Carvalho, J. Costa, and L. Wosinska, "Spectrum allocation policy modeling for elastic optical networks," presented at the High Capacity Optical Networks Enabling Technologies, Istanbul, Turkey, Dec. 2012.

[31] Y. Yin, H. Zhang, M. Zhang, M. Xia, Z. Zhu, S. Dahlfort, and S. Yoo, "Spectral and spatial 2D fragmentation-aware routing and spectrum assignment algorithms in elastic optical networks [invited]," J. Opt. Commun. Netw., vol. 5, no. 10, pp. A100-A106, Oct. 2013.

[32] P. Khodashenas, J. Comellas, S. Spadaro, J. Perello, and G. Junyent, "Using spectrum fragmentation to better allocate time-varying connections in elastic optical networks," J. Opt. Commun. Netw., vol. 6, no. 5, pp. 433-440, May 2014.
[33] A. Castro, L. Velasco, M. Ruiz, M. Klinkowski, J. Fernndez-Palacios, and D. Careglio, "Dynamic routing and spectrum (re)allocation in future flexgrid optical networks," Comput. Netw., vol. 56, no. 12, pp. 2869-2883, Dec. 2012.

[34] F. Paolucci, A. Castro, F. Fresi, M. Imran, A. Giorgetti, B. Bhownik, G. Berrettini, G. Meloni, F. Cugini, L. Velasco, L. Potì, and P. Castoldi, "Active PCE demonstration performing elastic operations and hitless defragmentation in flexible grid optical networks," Photon. Netw. Commun., vol. 29 , no. 1 , pp. 57-66, 2015.

[35] M. Dallaglio, A. Giorgetti, N. Sambo, F. Cugini, and P. Castoldi, "Impact of slice-ability on dynamic restoration in GMPLS-based flexible optical networks," presented at the Optical Fiber Communication Conf. Expo., Nat. Fiber Optic Engineers Conf., San Francisco, CA, USA, Mar. 2014.

[36] Z. Zhu, W. Lu, L. Zhang, and N. Ansari, "Dynamic service provisioning in elastic optical networks with hybrid single-/multi-path routing," J. Lightw. Technol., vol. 31, no. 1, pp. 15-22, Jan. 2013.

[37] A. Pages, J. Perello, S. Spadaro, and J. Comellas, "Optimal route, spectrum, and modulation level assignment in split-spectrum-enabled dynamic elastic optical networks," J. Opt. Commun. Netw., vol. 6, no. 2, pp. 114 126, Feb. 2014

[38] A. Pages, J. Perello, and S. Spadaro, "Lightpath fragmentation for efficient spectrum utilization in dynamic elastic optical networks," presented at the 16th Int. Conf. Optical Network Design Modeling, Colchester, U.K., Apr. 2012.

[39] M. Dallaglio, A. Giorgetti, N. Sambo, and P. Castoldi, "Impact of sbvts based on multi-wavelength source during provisioning and restoration in elastic optical networks," presented at the Optical Fiber Communication Conf. Expo., Nat. Fiber Optic Engineers Conf., Cannes, France, Sep. 2014

[40] R. Munoz, R. Vilalta, R. Casellas, R. Martinez, S. Frigerio, and A. Lometti, "Design and experimental evaluation of dynamic inverse-multiplexing provisioning in GMPLS-controlled flexi-grid DWDM networks with sliceable OTN BVTs," presented at the Optical Fiber Communication Conf. Expo., Nat. Fiber Optic Engineers Conf., London, U.K., Sep. 2013.

[41] J. Santos, J. Pedro, P. Monteiro, and J. Pires, "Optimized routing and buffer design for optical transport networks based on virtual concatenation," $J$. Opt. Commun. Netw., vol. 3, no. 9, pp. 725-738, Sep. 2011.

[42] J. Santos, J. Pedro, A. Eira, P. Monteiro, and J. Pires, "Optical transport network design with collocated regeneration and differential delay compensation," in Proc. IEEE 13th Int. Conf. High Perform. Switching Routing, Jun. 2012, pp. 204-209.

[43] S. Poole, S. Frisken, M. Roelens, and C. Cameron, "Bandwidth-flexible ROADMs as network elements," presented at the Optical Fiber Communication Conf. Expo., Nat. Fiber Optic Engineers Conf., Los Angeles, CA, USA, Mar. 2011.

[44] J. Zhang, Y. Ji, M. Song, Y. Zhao, X. Yu, J. Zhang, and B. Mukherjee, "Dynamic traffic grooming in sliceable bandwidth-variable transponderenabled elastic optical networks," J. Lightw. Technol., vol. 33, no. 1, pp. 183-191, Jan. 2015.

[45] F. Paolucci, F. Cugini, A. Giorgetti, N. Sambo, and P. Castoldi, "A survey on the path computation element (PCE) architecture," IEEE Commun. Surveys Tuts., vol. 15, no. 4, pp. 1819-1841, Fourth Quarter 2013.

[46] A. Giorgetti, F. Cugini, N. Sambo, F. Paolucci, N. Andriolli, and P. Castoldi, "Path state-based update of PCE traffic engineering database in wavelength switched optical networks," IEEE Commun. Lett., vol. 14, no. 6, pp. 575-577, Jun. 2010.

[47] OPNET Modeler suite provided by Riverbed. (2000). [Online]. Available: http://www.riverbed.com/products/performance-managementcontrol/opnet.html.

Authors' biographies not available at the time of publication. 